Tropical Journal of Pharmaceutical Research April 2011; 10 (2): 219-229

(C) Pharmacotherapy Group,

Faculty of Pharmacy, University of Benin

Benin City, 300001 Nigeria.

All rights reserved.

Research Article

Available online at http://www.tjpr.org

\title{
Synthesis, Antimicrobial and Antitubercular Activities of Some Novel Trihydroxy Benzamido Azetidin-2-one Derivatives
}

\section{K Ilango* and S Arunkumar}

Department of Pharmaceutical Chemistry, College of Pharmacy, SRM University, Kattankulthur - 603203, Tamilnadu, India

\begin{abstract}
Purpose: To synthesize and characterize novel trihydroxy benzamido azetidin-2-one derivatives and screen them for antimicrobial and antitubercular activities.

Methods: A series of novel 4-aryl-3-chloro-N-(3,4,5-trihydroxy benzamido)-2-azetidinones, 3a-o, were synthesized by reacting various Schiff bases of galloyl hydrazide, 2a-0, with chloroacetyl chloride in the presence of dioxan and triethylamine. Schiff bases of galloyl hydrazide, 2a-o, were synthesized from galloyl hydrazide. The newly synthesized compounds were characterized by infrared spectroscopy (IR), mass spectroscopy (MS) and proton nuclear magnetic spectroscopy ${ }^{1} H$ NMR) and elemental analysis; they were also screened for in vitro antibacterial, antifungal and antitubercular activities. Ciprofloxacin and ketoconazole were used as reference standards for antibacterial and antifungal activities, respectively, while isoniazid was used as reference standard for antitubercular activity.

Results: Compounds $\mathbf{3 f}, \mathbf{3} \mathbf{g}$ and $\mathbf{3 o}$ with chlorophenyl group and compound $\mathbf{3 k}$ with 4-dimethyl amino phenyl group exhibited good antimicrobial activity. Also, compounds $\mathbf{3 f}, \mathbf{3 g}, \mathbf{3 k}$ and $\mathbf{3 o}$ showed antitubercular activity with minimum inhibitory concentration (MIC) values equivalent to the standard drug (isoniazid). MIC values of $\mathbf{3 f}$, $\mathbf{3 g}, \mathbf{3 k}$ and $\mathbf{3 o}$ were $0.76,0.57 .0 .62$ and $0.83 \mu \mathrm{g} / \mathrm{ml}$, respectively, while the MIC of isoniazid was 0.56 .

Conclusion: We report the successful synthesis, spectral characterization, as well as in vitro antimicrobial and antitubercular evaluation of a series of novel trihydroxy benzamido azetidin-2-one derivatives. The work shows the emergence of new antimicrobial and antitubercular compounds.
\end{abstract}

Keywords: Azetidinone, Schiff bases, Synthesis, Antimicrobial activity, Antitubercular activity. 


\section{INTRODUCTION}

Tuberculosis, one of the most common infections, is caused by Mycobacterium tuberculosis. According to the World Health Organization (WHO), nearly one third of the world's population has been exposed to the tuberculosis pathogen [1]. Annually, 8 million people become ill with tuberculosis and 2 million people die from the disease worldwide. Every 15 seconds, someone dies of tuberculosis [2]. There are a number of known factors that make people more susceptible to tuberculosis infection worldwide, the most important of which is human immunodeficiency virus (HIV). The association of tuberculosis with HIV infection is so dramatic that in some cases, nearly twothird of the patients diagnosed with the tuberculosis are also HIV-1 seropositive [3]. Smoking more than 20 cigarettes a day also increases the risk of tuberculosis by two- to four times [4].

Following the discovery of isoniazid, there have been no new classes of antitubercular drugs in past 40 years [5]. Moreover, there has been a recent and disturbing increase in the number of tubercular cases that are caused by organisms which are resistant to the first-line drugs such as isoniazid, rifampicin, ethambutol, streptomycin and pyrazinamide [6]. The Global Alliance for Tuberculosis (GATB) drug development was established to address this need [7]. Its top priority is the development of a new agent that will shorten the duration of chemotherapy from the current $6-8$ months to two months or less. Also new drugs with activity against mycobacterium drug-resistant tuberculosis and latent tuberculosis are needed.

The addition of a beta-lactamase inhibitor to amoxicillin greatly improves its in vitro activity against $M$. tuberculosis [8]. Gallic acid is a naturally existing antioxidant [9] which occurs along with tannins in most plant species. It possesses various biological activities such as analgesic [10], antimicrobial [11], antineoplastic [12] and antityrosinase [13].

Azetidinone is chemically 2Azacyclobutanone which is an integral part of penicillin. Ezetimibe, an azetidinone, represents the first new approach to lipid lowering therapy in more than a decade [14]. Moreover, this heterocycle is associated with muscle relaxant [15], antitubercular [16] and antiinflammatory [17] activities. Considering the above pharmacological activities of both gallic acid and azetidinones we thought that it would be worthwhile to incorporate the azetidinone ring into gallic acid to have synergestic effect.

\section{EXPERIMENTAL}

\section{Equipment}

Reactions were monitored by thin layer chromatography (TLC) on precoated silica gel plates obtained from E. Merck and Co. Melting points were determined using Veego digital melting point apparatus and are uncorrected. IR spectra were recorded on a Perkin Elmer FTIR Spectrometer. ${ }^{1} \mathrm{H}$ NMR spectra were measured with a Bruker Spectrometer $[400 \mathrm{MHz}]$ in $\mathrm{CDCl}_{3}$ using tetramethylsilane (TMS) TMS as an internal standard. Mass spectra were obtained with LC-MSD Trap- SL 2010A Shimadzu. Elemental analysis was determined on a Perkin Elmer - $240 \mathrm{CHN}$ elemental analyzer.

\section{Materials}

All the chemicals used were of analytical reagent grade and obtained from Ranbaxy Fine Chemicals Ltd, India. Muller-Hinton and Sabouraud dextrose agars were obtained from Hi-Media Ltd, India. The bacterial strains were provided by School of Biotechnology, SRM University, Kattankulathur, India. Ciprofloxacin and ketaconazole were obtained from M/S Orchid Chemicals and Pharmaceuticals Limited, Chennai, India. 
Ilango \& Arunkumar
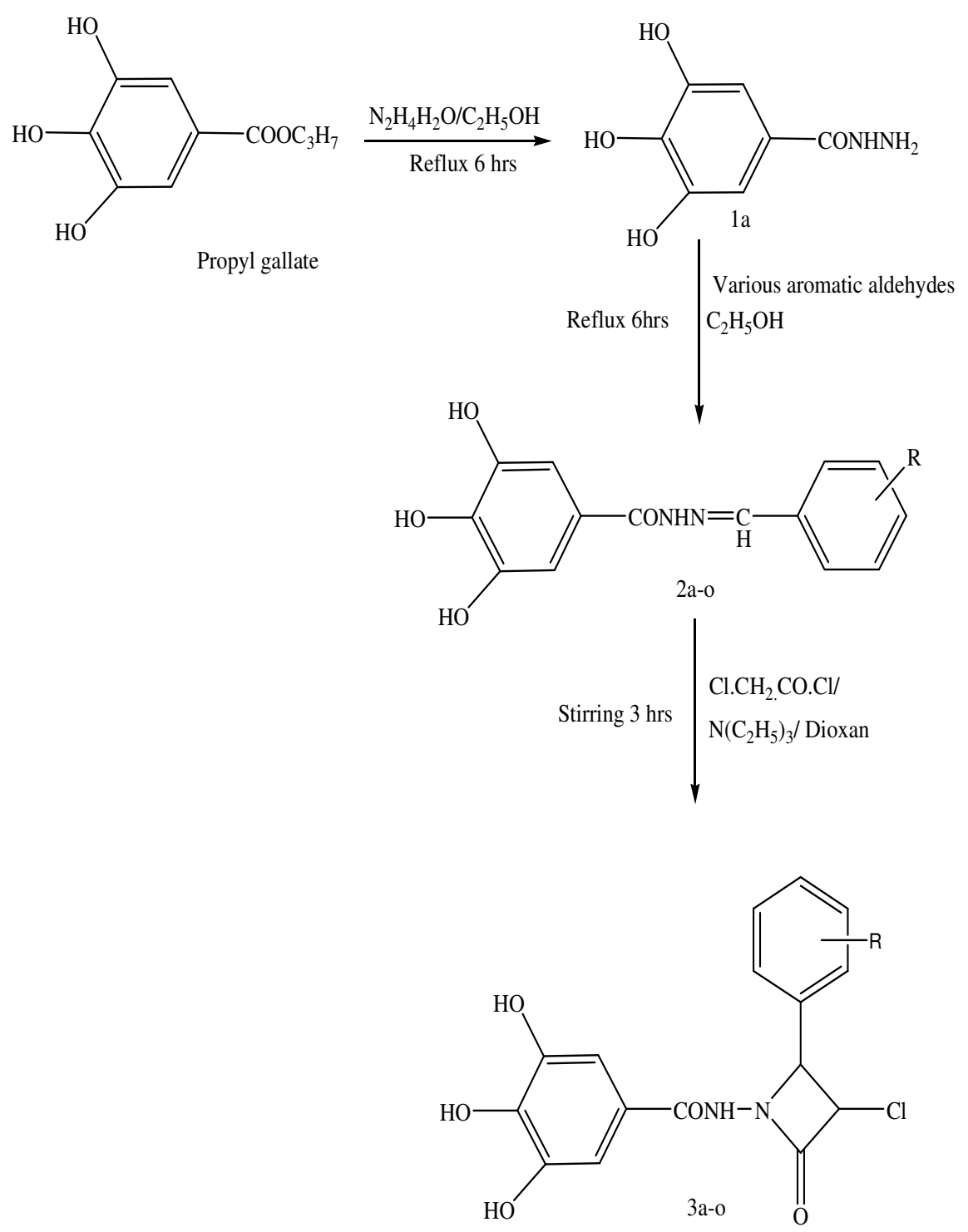

Fig 1: Synthesis of novel trihydroxybenzamido azetidin-2-one derivatives

\section{Methods}

All the compounds were synthesized according to the scheme shown in Figure 1. The purity of the compounds was determined on TLC plates using silica gel $G$ as a stationary phase and iodine vapors as visualizing agent.
Synthesis of 3,4,5-trihydroxy benzoic acid hydrazide from propyl gallate (1a)

Propyl gallate $(0.01 \mathrm{~mol})$ in ethanol and hydrazine hydrate $(0.01 \mathrm{~mol})$ were refluxed for $6 \mathrm{~h}$. The excess of solvent was distilled off under reduced pressure using a vacuum pump. The cold residual mass was washed with distilled water, filtered and dried. The 
crude product obtained was recrystallised from methanol to yield galloyl hydrazide 1a. Yield: $85 \%$, Colour: Pale white, $R_{f}$ value: $0.61, \mathrm{Mp}: 167^{\circ} \mathrm{C}$.

General procedure for synthesis of $\mathrm{N}$ substituted arylidene galloyl hydrazide (2a-o)

Equimolar quantities of galloyl hydrazide 1a and various aromatic aldehydes in ethanol were refluxed for $6 \mathrm{~h}$. The completion of reaction was monitored on silica gel $G$ precoated TLC plates using hexane and ethyl acetate $(1: 1)$ as an eluent and observed under UV light. The resultant mixture was poured into ice cold water. The crude Schiff bases were washed, filtered, dried and recrystallised from ethanol.

N'--benzylidene galloyl hydrazide (2a): Yield: $73 \%$, Colour: Pale white, $R_{f}$ value: 0.61,M.p.; 170-172 ${ }^{\circ} \mathrm{C}$. IR $\left(\mathrm{KBr}\right.$ in $\left.\mathrm{cm}^{-1}\right): 3407$ (NH Str); 3313 (Ar-OH Str); 2987 (Ar-CH Str); 1693 (C=O Str); 1480 (C=N Str); 1446 (CO$\mathrm{NH}$ Str). ${ }^{1} \mathrm{H}$ NMR (DMSO, $\left.\delta \mathrm{ppm}\right): 8.35$ (s, $1 \mathrm{H}, \mathrm{CH}=\mathrm{N}), 7.28-7.95(\mathrm{~m}, 7 \mathrm{H}, \mathrm{Ar}-\mathrm{H}), 4.34$ (s, $1 \mathrm{H}, \mathrm{NH}), 2.83(\mathrm{~s}, 3 \mathrm{H}, 3(\mathrm{OH}))$.

\section{N'- (2-hydroxy) benzylidene galloyl} hydrazide (2b): Yield: 65\%, Colour: Pale yellow, $R_{f}$ value: 0.61, M.p.; 168-169 ${ }^{\circ} \mathrm{C}$. IR $\left(\mathrm{KBr}\right.$ in $\mathrm{cm}^{-1}$ ): 3411 (NH Str); 3332 (Ar-OH Str); 2971 (Ar-CH Str); 1675 (C=O Str); 1476 (C=N Str); 1438 (CO-NH Str). ${ }^{1} \mathrm{H}$ NMR (DMSO, $\delta \mathrm{ppm}$ ): $8.37(\mathrm{~s}, 1 \mathrm{H}, \mathrm{CH}=\mathrm{N}), 6.96-$ $7.95(\mathrm{~m}, 6 \mathrm{H}, \mathrm{Ar}-\mathrm{H}), 4.31(\mathrm{~s}, 1 \mathrm{H}, \mathrm{NH}), 2.76(\mathrm{~s}$, $4 \mathrm{H}, 4(\mathrm{OH}))$.

N'- (2-hydroxy-3-methoxy) benzylidene galloyl hydrazide (2c): Yield: $77 \%$, Colour: Pale white, $R_{f}$ value: 0.61 , M.p.; 171-172 ${ }^{\circ} \mathrm{C}$. IR ( $\mathrm{KBr}$ in $\left.\mathrm{cm}^{-1}\right)$ : 3331 (NH Str); 3310 (Ar-OH Str); 2994 (Ar-CH Str); 1678 (C=O Str); 1480 (C=N Str); 1444(CO-NH Str); 1264 (C-O-C Str). ${ }^{1} \mathrm{H}$ NMR (DMSO, $\left.\delta \mathrm{ppm}\right):$ 8.25-9.12 (m, $5 \mathrm{H}, \mathrm{Ar}-\mathrm{H}), 8.41$ (s, $1 \mathrm{H}, \mathrm{CH}=\mathrm{N}), 4.98(\mathrm{~s}, 1 \mathrm{H}$, $\mathrm{NH}), 3.9\left(\mathrm{~s}, 3 \mathrm{H}, \mathrm{Ar}-\mathrm{OCH}_{3}\right), 3.48(\mathrm{~s}, 4 \mathrm{H}$, $4(\mathrm{OH}))$.
N'- (3-hydroxy) benzylidene galloyl hydrazide (2d): Yield: $79 \%$, Colour: Pale brown, $R_{f}$ value: 0.61 , M.p.; $170-171{ }^{\circ} \mathrm{C}$. IR $\left(\mathrm{KBr}\right.$ in $\left.\mathrm{cm}^{-1}\right)$ : 3334 (NH Str); 3312 (Ar-OH Str); 2981 (Ar-CH Str); 1672 (C=O Str); 1470 ( $\mathrm{C}=\mathrm{N}$ Str); 1442 (CO-NH Str). ${ }^{1} \mathrm{H}$ NMR (DMSO, $\delta \mathrm{ppm})$ : 8.91-9.52 (m, 6H, Ar-H), $8.37(\mathrm{~s}, 1 \mathrm{H}, \mathrm{CH}=\mathrm{N}), 4.41(\mathrm{~s}, 1 \mathrm{H}, \mathrm{NH}), 3.67$ (s, $4 \mathrm{H}, 4(\mathrm{OH}))$.

N'- (4-hydroxy) benzylidene galloyl hydrazide (2e): Yield: $82 \%$, Colour: Pale white, $\mathrm{R}_{\mathrm{f}}$ value: 0.61 , M.p.; 172-174 ${ }^{\circ} \mathrm{C}$. IR $\left(\mathrm{KBr}\right.$ in $\mathrm{cm}^{-1}$ ): 3318 (NH Str); 3309 (Ar-OH Str); 2973 (Ar-CH Str); 1674 (C=O Str); 1476 $\left(\mathrm{C}=\mathrm{N}\right.$ Str); 1438 (CO-NH Str). ${ }^{1} \mathrm{H}$ NMR (DMSO, $\delta \mathrm{ppm})$ : 8.48-9.07 (m, 6H, Ar-H), $8.34(\mathrm{~s}, 1 \mathrm{H}, \mathrm{CH}=\mathrm{N}), 4.4(\mathrm{~s}, 1 \mathrm{H}, \mathrm{NH}), 2.38(\mathrm{~s}$, $4 \mathrm{H}, 4(\mathrm{OH}))$.

N'- (2-chloro) benzylidene galloyl hydrazide (2f): Yield: $79 \%$, Colour: Pale white, $\mathrm{R}_{\mathrm{f}}$ value: 0.61 , M.p.; 168-170 ${ }^{\circ} \mathrm{C}$. IR $\left(\mathrm{KBr}\right.$ in $\mathrm{cm}^{-1}$ ): 3410 (NH Str); 3304 (Ar-OH Str); 2994 (Ar-CH Str); 1758 (C=O Str); 1438 (C=N Str); 1419 (CO-NH Str); 762 (C-Cl Str). ${ }^{H} \mathrm{H}$ NMR (DMSO, $\left.\delta \mathrm{ppm}\right):$ 8.54-9.67 $(\mathrm{m}, 5 \mathrm{H}$, Ar-H), $8.36(\mathrm{~s}, 1 \mathrm{H}, \mathrm{CH}=\mathrm{N}), 7.73(\mathrm{~s}, 1 \mathrm{H}, \mathrm{CH}-$ $\mathrm{Cl}), 4.28(\mathrm{~s}, 1 \mathrm{H}, \mathrm{NH}), 2.79(\mathrm{~s}, 3 \mathrm{H}, 3(\mathrm{OH}))$.

N'- (3-chloro) benzylidene galloyl hydrazide (2g): Yield: $81 \%$, Colour: Pale yellow, $\mathbf{R}_{\mathbf{f}}$ value: 0.61 , M.p.; 171-173으. IR $\left(\mathrm{KBr}\right.$ in $\left.\mathrm{cm}^{-1}\right)$ : 3335 (NH Str); 3318 (Ar-OH Str); 2983 (Ar-CH Str); 1758 (C=O Str); 1437 (C=N Str); 1422 (CO-NH Str); 760 (C-Cl Str). ${ }^{1} \mathrm{H}$ NMR (DMSO, $\left.\delta \mathrm{ppm}\right): 8.32(\mathrm{~s}, 1 \mathrm{H}$, $\mathrm{CH}=\mathrm{N}), 7.48-8.52(\mathrm{~m}, 5 \mathrm{H}, \mathrm{Ar}-\mathrm{H}), 7.64(\mathrm{~s}, 1 \mathrm{H}$, $\mathrm{CH}-\mathrm{Cl}), 4.38(\mathrm{~s}, 1 \mathrm{H}, \mathrm{NH}), 2.92(\mathrm{~s}, 3 \mathrm{H}$, $3(\mathrm{OH}))$.

N'- (2-nitro) benzylidene galloyl hydrazide (2h): Yield: $80 \%$, Colour: Pale brown, $R_{f}$ value: 0.61 , M.p.; $170-171^{\circ} \mathrm{C}$. IR ( $\mathrm{KBr}$ in $\mathrm{cm}^{-1}$ ): 3328 (NH Str); 3302 (Ar-OH Str); 2974 (Ar-CH Str); 1749 (C=O Str); 1534 (C-NO Str); 1441 (C=N Str); 1421 (CO-NH Str). ${ }^{1} \mathrm{H}$ NMR (DMSO, $\delta$ ppm): 7.23-8.51 (m, 6H, Ar$\mathrm{H}), 8.31(\mathrm{~s}, 1 \mathrm{H}, \mathrm{CH}=\mathrm{N}), 4.49(\mathrm{~s}, 1 \mathrm{H}, \mathrm{NH})$, $2.63(\mathrm{~s}, 3 \mathrm{H}, 3(\mathrm{OH}))$. 
N'- (3-nitro) benzylidene galloyl hydrazide (2i): Yield: $72 \%$, Colour: Pale yellow, $R_{f}$ value: 0.61 , M.p.; $171-172^{\circ} \mathrm{C}$. IR $\left(\mathrm{KBr}\right.$ in $\mathrm{cm}^{-}$ 1): 3400 (NH Str); 3312 (Ar-OH Str); 2971 (Ar$\mathrm{CH}$ Str); 1685 (C=O Str); 1535 (C-NO 2 Str); 1480 (C=N Str); 1437 (CO-NH Str). ${ }^{1} \mathrm{H}$ NMR (DMSO, $\delta \mathrm{ppm})$ : 7.41-8.69 (m, 6H, Ar-H), $8.41(\mathrm{~s}, 1 \mathrm{H}, \mathrm{CH}=\mathrm{N}), 4.52(\mathrm{~s}, 1 \mathrm{H}, \mathrm{NH}), 2.56(\mathrm{~s}$, $3 \mathrm{H}, 3(\mathrm{OH}))$.

N'- (4-nitro) benzylidene galloyl hydrazide (2j): Yield: $85 \%$, Colour: Pale brown, $R_{f}$ value: 0.61 , M.p.; $171-172^{\circ} \mathrm{C}$. IR $\left(\mathrm{KBr}\right.$ in $\mathrm{cm}^{-}$ $\left.{ }^{1}\right)$ : 3405 (NH Str); 3315 (Ar- OH Str); 2987 (Ar-CH Str); 1685 (C=O Str); 1538 (C-NO Str); 1480 (C=N Str); 1432 (CO-NH Str). ${ }^{1} \mathrm{H}$ NMR (DMSO, $\delta \mathrm{ppm}):$ 8.44-8.91 (m, 6H, Ar$\mathrm{H}), 8.34(\mathrm{~s}, 1 \mathrm{H}, \mathrm{CH}=\mathrm{N}), 4.52(\mathrm{~s}, 1 \mathrm{H}, \mathrm{NH})$, $2.67(\mathrm{~s}, 3 \mathrm{H}, 3(\mathrm{OH}))$.

N'- (4-dimethylamino) benzylidene galloyl hydrazide (2k): Yield: $77 \%$, Colour: Pale white, $\mathrm{R}_{\mathrm{f}}$ value: 0.61 , M.p.; 175-178 ${ }^{\circ} \mathrm{C}$. IR $\left(\mathrm{KBr}\right.$ in $\mathrm{cm}^{-1}$ ): 3395 (NH Str); 3331 (Ar- OH Str); 2997 (Ar-CH Str); 1668 (C=O Str); 1535 $\left(\mathrm{C}-\mathrm{NO}_{2} \mathrm{Str}\right) ; 1410$ (C=N Str); 1441 (CO-NH Str). ${ }^{1} \mathrm{H}$ NMR (DMSO, $\left.\delta \mathrm{ppm}\right)$ : 8.31-9.33 (m, $6 \mathrm{H}, \mathrm{Ar}-\mathrm{H}), 8.42(\mathrm{~s}, 1 \mathrm{H}, \mathrm{CH}=\mathrm{N}), 4.53(\mathrm{~s}, 1 \mathrm{H}$, $\mathrm{NH}), 2.21(\mathrm{~s}, 3 \mathrm{H}, 3(\mathrm{OH})), 2.19(\mathrm{~s}, 6 \mathrm{H}$, $\left.\mathrm{N}\left(\mathrm{CH}_{3}\right)_{2}\right)$.

N'- (3,4,5-trimethoxy) benzylidene galloyl hydrazide (2l): Yield: $81 \%$, Colour: Pale yellow, $R_{f}$ value: 0.61 , M.p.; 171-173 ${ }^{\circ} \mathrm{C}$. IR $\left(\mathrm{KBr}\right.$ in $\mathrm{cm}^{-1}$ ): 3397 (NH Str); 3313 (Ar-OH Str); 2977 (Ar-CH Str); 1687 (C=O Str); 1617 (C-O-C Str); 1480 (C=N Str); 1445 (CO-NH Str). ${ }^{1} \mathrm{H}$ NMR (DMSO, $\left.\delta \mathrm{ppm}\right):$ 8.18-8.85 (m, $4 \mathrm{H}, \mathrm{Ar}-\mathrm{H}), 8.28(\mathrm{~s}, 1 \mathrm{H}, \mathrm{CH}=\mathrm{N}), 4.89(\mathrm{~s}, 1 \mathrm{H}$, $\mathrm{NH}), 3.62\left(\mathrm{~s}, 9 \mathrm{H},\left(\mathrm{OCH}_{3}\right)_{3}\right), 2.71(\mathrm{~s}, 3 \mathrm{H}$, $3(\mathrm{OH}))$.

N'- (3,4,-dimethoxy) benzylidene galloyl hydrazide (2m): Yield: $82 \%$, Colour: Pale browm, $\mathrm{R}_{\mathrm{f}}$ value: 0.61, M.p.; 168-169․ $\mathrm{C}$. IR $\left(\mathrm{KBr}\right.$ in $\mathrm{cm}^{-1}$ ): 3405 (NH Str); 3333 (Ar-OH Str); 2987 (Ar-CH Str); 1670 (C=O Str); 1614 (C-O-C Str); 1475 (C=N Str); 1439 (CO-NH Str). ${ }^{1} \mathrm{H}$ NMR (DMSO, $\left.\delta \mathrm{ppm}\right):$ 8.16-9.12 (m, $5 \mathrm{H}, \mathrm{Ar}-\mathrm{H}), 8.37(\mathrm{~s}, 1 \mathrm{H}, \mathrm{CH}=\mathrm{N}), 4.71(\mathrm{~s}, 1 \mathrm{H}$,
$\mathrm{NH}), 3.82\left(\mathrm{~s}, 6 \mathrm{H},\left(\mathrm{OCH}_{3}\right)_{2}\right), 2.41(\mathrm{~s}, 3 \mathrm{H}$, $3(\mathrm{OH}))$.

N'- (4-methoxy) benzylidene galloyl hydrazide (2n): Yield: $87 \%$, Colour: Pale yellow, $R_{f}$ value: 0.61 , M.p.; 171-173으. IR $\left(\mathrm{KBr}\right.$ in $\left.\mathrm{cm}^{-1}\right)$ : 3405 (NH Str); 3333 (Ar-OH Str); 2972 (Ar-CH Str); 1670 (C=O Str); 1614 (C-O-C Str); 1475 (C=N Str); 1433 (CO-NH Str). ${ }^{1} \mathrm{H}$ NMR (DMSO, $\left.\delta \mathrm{ppm}\right):$ 8.54-9.77 (m, $6 \mathrm{H}, \mathrm{Ar}-\mathrm{H}), 8.36(\mathrm{~s}, 1 \mathrm{H}, \mathrm{CH}=\mathrm{N}), 4.18(\mathrm{~s}, 1 \mathrm{H}$, $\mathrm{NH}), 3.82\left(\mathrm{~s}, 3 \mathrm{H}, \mathrm{OCH}_{3}\right), 2.74(\mathrm{~s}, 3 \mathrm{H}, 3(\mathrm{OH}))$.

N'-(4-chloro) benzylidene galloyl hydrazide (20): Yield: $83 \%$, Colour: Pale white, $R_{f}$ value: 0.61 , M.p.; $179-181^{\circ} \mathrm{C}$. IR $\left(\mathrm{KBr}\right.$ in $\mathrm{cm}^{-}$ 1): 3405 (NH Str); 3328 (Ar-OH Str); 2976 (ArCH Str); 1666 (C=O Str); 1624 (C-O-C Str); 1435 (C=N Str); 1424 (CO-NH Str); 763 (C-Cl Str). ${ }^{1} \mathrm{H}$ NMR (DMSO, $\left.\delta \mathrm{ppm}\right):$ 8.39-8.95 (m, $5 \mathrm{H}, \mathrm{Ar}-\mathrm{H}), 8.36(\mathrm{~s}, 1 \mathrm{H}, \mathrm{CH}=\mathrm{N}), 7.7$ (s, $1 \mathrm{H}$, $\mathrm{CH}-\mathrm{Cl}), 4.15(\mathrm{~s}, 1 \mathrm{H}, \mathrm{NH}), 2.14(\mathrm{~s}, 3 \mathrm{H}$, $3(\mathrm{OH}))$.

General procedure for Synthesis 4-Aryl-3chloro- $\mathrm{N}-(3,4,5$-trihydroxy benzamido)-2azetidinones (3a-0)

A mixture of Schiff base $(0.002 \mathrm{~mol})$ and triethyl amine $(0.004 \mathrm{~mol})$ were dissolved in 1,4-Dioxan $(50 \mathrm{ml})$. To this, well stirred cold solution of chloroacetyl chloride $(0.004 \mathrm{~mol})$ was added drop wise for $20 \mathrm{~min}$, then stirred for further $3 \mathrm{~h}$ and left at room temperature for $48 \mathrm{~h}$ [18]. The resultant mixture was concentrated, cooled and then poured into ice cold water filtered off, dried and recrystalised from methanol.

(3R, 4S)-3-Chloro-4-(phenyl)-N-(-3,4,5trihydroxy benzamido)-2-azetidinone (3a):

Yield: $70 \%$, Colour: Dull white, $R_{f}$ value: 0.63, M.p.; $221^{\circ} \mathrm{C}$. Anal. calcd.for $\mathrm{C}_{16} \mathrm{H}_{13} \mathrm{ClN}_{2} \mathrm{O}_{5}$ (348): C, 55.10; $\mathrm{H}, 3.76 ; \mathrm{N}$, $8.03 \%$. Found: C, 55.17; H, 3.68; N, $7.98 \%$. IR ( $\mathrm{KBr}$ in $\left.\mathrm{cm}^{-1}\right): 3613$ (Ar-OH Str); 3467 (NH Str); 2977 (Ar-CH Str); 1744 (C=O Str); 1648 (CO-NH Str); 1474 (CH-N Str); 1446 (CO-NH Str); 759 (C-Cl Str). ${ }^{1} \mathrm{H}$ NMR (DMSO, $\delta$ ppm): $7.7(\mathrm{~s}, 1 \mathrm{H}, \mathrm{CH}-\mathrm{Cl}), 7.08-7.44(\mathrm{~m}, 7 \mathrm{H}, \mathrm{Ar}-\mathrm{H})$, 
$5.2(\mathrm{~s}, 1 \mathrm{H}, \mathrm{CH}-\mathrm{N}), 4.15(\mathrm{~s}, 1 \mathrm{H}, \mathrm{NH}), 2.33(\mathrm{~s}$, $3 \mathrm{H}, 3(\mathrm{OH}))$. MS (m/z): $349\left(\mathrm{M}^{+1}\right)$.

(3R, 4S)-3-Chloro-4-(2-hydroxy phenyl)-N(-3,4,5-trihydroxy benzamido)-2azetidinone (3b): Yield: $73 \%$, Colour: Pale yellow, $R_{f}$ value: 0.78, M.p.; $198^{\circ} \mathrm{C}$. Anal. calcd.for $\mathrm{C}_{16} \mathrm{H}_{13} \mathrm{ClN}_{2} \mathrm{O}_{6}$ (364): C, 54.34; $\mathrm{H}$, 7.68; N, $6.67 \%$. Found: C, 54.32; H,7.65; $\mathrm{N}, 6.65 \%$. IR ( $\mathrm{KBr}$ in $\left.\mathrm{cm}^{-1}\right): 3613$ (Ar-OH Str); 3467 (NH Str); 2977 (Ar-CH Str); 1755 (C=O Str); 1648 (CONH Str); 1474 (CH-N Str); 1446 (CO-NH Str); 757 (C-Cl Str). ${ }^{1} \mathrm{H}$ NMR (DMSO, $\delta \mathrm{ppm})$ : 7.68-8.34 (m, 6H, Ar-H), $7.65(\mathrm{~s}, 1 \mathrm{H}, \mathrm{CH}-\mathrm{Cl}), 5.43(\mathrm{~s}, 1 \mathrm{H}, \mathrm{CH}-\mathrm{N}), 4.75$ (s, $1 \mathrm{H}, \mathrm{NH}), 2.76(\mathrm{~s}, 4 \mathrm{H}, 4(\mathrm{OH})) . \mathrm{MS}(\mathrm{m} / \mathrm{z})$ : $365\left(\mathrm{M}^{+1}\right)$.

(3R, 4S)-3-Chloro-4-(2-hydroxy 3-methoxy phenyl)-N-(-3,4,5-trihydroxy benzamido)-2azetidinone (3c): Yield: $67 \%$, Colour: White, $\mathrm{R}_{\mathrm{f}}$ value: 0.72 , M.p.; $217^{\circ} \mathrm{C}$. Anal. calcd. for $\mathrm{C}_{17} \mathrm{H}_{15} \mathrm{CIN}_{2} \mathrm{O}_{7}$ (378): C, 53.39; $\mathrm{H}, 7.62 ; \mathrm{N}$, $6.23 \%$. Found: C, 53.37; H, 7.60; N, 6.28\%. IR (KBr in cm $\left.{ }^{-1}\right): 3614$ (Ar-OH Str); $3458(\mathrm{NH}$ Str); 2985 (Ar-CH Str); 1743 (C=O Str); 1661 (CONH Str); 1482 (CH-N Str); 1446 (CO-NH Str); 1268 (C-O-C Str); 762 (C-Cl Str). ${ }^{1} \mathrm{H}$ NMR (DMSO, $\delta$ ppm): 7.12- $8.44(\mathrm{~m}, 5 \mathrm{H}, \mathrm{Ar}-$ $\mathrm{H}), 7.7$ (s, 1H, CH-Cl), $5.5(\mathrm{~s}, 1 \mathrm{H}, \mathrm{CH}-\mathrm{N}), 4.4$ (s, $1 \mathrm{H}, \mathrm{NH}), 3.9\left(\mathrm{~s}, 3 \mathrm{H}, \mathrm{OCH}_{3}\right), 3.48(\mathrm{~s}, 4 \mathrm{H}$, $4(\mathrm{OH}))$. MS (m/z): $379\left(\mathrm{M}^{+1}\right)$.

(3R, 4S)-3-Chloro-4-(3-hydroxy phenyl)-N(-3,4,5-trihydroxy benzamido)-2azetidinone (3d): Yield: $63 \%$, Colour: White, $R_{f}$ value: 0.69 , M.p.; $206{ }^{\circ} \mathrm{C}$. Anal. calcd. for $\mathrm{C}_{16} \mathrm{H}_{13} \mathrm{CIN}_{2} \mathrm{O}_{6}$ (364): C, 54.34; $\mathrm{H}$, $7.68 ; \mathrm{N}, 6.67 \%$. Found: C, 54.28; $\mathrm{H}, 7.59 ; \mathrm{N}$, $6.79 \%$. IR ( $\mathrm{KBr}$ in $\left.\mathrm{cm}^{-1}\right): 3632$ (Ar-OH Str); 3465 (NH Str); 2977 (Ar-CH Str); 1733 (C=O Str); 1667 (CONH Str); 1458 (CH-N Str); 1446 (CO-NH Str); 752 (C-Cl Str). ${ }^{1} \mathrm{H}$ NMR (DMSO, $\delta \mathrm{ppm}):$ 7.52- $8.91(\mathrm{~m}, 6 \mathrm{H}, \mathrm{Ar}-\mathrm{H})$, $7.48(\mathrm{~s}, 1 \mathrm{H}, \mathrm{CH}-\mathrm{Cl}), 5.5(\mathrm{~s}, 1 \mathrm{H}, \mathrm{CH}-\mathrm{N}), 4.4(\mathrm{~s}$, $1 \mathrm{H}, \mathrm{NH}), 3.31(\mathrm{~s}, 4 \mathrm{H}, 4(\mathrm{OH}))$. MS (m/z): 365 $\left(\mathrm{M}^{+1}\right)$.

(3R, 4S)-3-Chloro-4-(4-hydroxy phenyl)-N(-3,4,5-trihydroxy benzamido)-2- azetidinone (3e): Yield: $64 \%$, Colour: Pale yellow, $R_{f}$ value: 0.83 , M.p.; $228{ }^{\circ} \mathrm{C}$. Anal. calcd. for $\mathrm{C}_{16} \mathrm{H}_{13} \mathrm{CIN}_{2} \mathrm{O}_{6}$ (364): C, 54.34; $\mathrm{H}, 7.68$; N, 6.67\%. Found: C, 54.38; H, 7.65; $\mathrm{N}, 6.69 \%$. IR $\left(\mathrm{KBr}\right.$ in $\left.\mathrm{cm}^{-1}\right): 3609$ (Ar-OH Str); 3358 (NH Str); 2978 (Ar-CH Str); 1772 $(\mathrm{C}=\mathrm{O} \mathrm{Str}) ; 1653$ (CONH Str); 1483 (CH-N Str); 758 (C-Cl Str). ${ }^{1} \mathrm{H}$ NMR (DMSO, $\delta$ ppm): 7.08- $8.58(\mathrm{~m}, 6 \mathrm{H}, \mathrm{Ar}-\mathrm{H}), 7.7(\mathrm{~s}, 1 \mathrm{H}, \mathrm{CH}-\mathrm{Cl})$, $5.5(\mathrm{~s}, 1 \mathrm{H}, \mathrm{CH}-\mathrm{N}), 4.4(\mathrm{~s}, 1 \mathrm{H}, \mathrm{NH}), 2.78(\mathrm{~s}$, $4 \mathrm{H}, 4(\mathrm{OH})) . \mathrm{MS}(\mathrm{m} / \mathrm{z}): 365\left(\mathrm{M}^{+1}\right)$.

(3R, 4S)-3-Chloro-4-(2-chloro phenyl)-N-(3,4,5-trihydroxy benzamido)-2-azetidinone (3f): Yield: $71 \%$, Colour: Light brown, $R_{f}$ value: 0.56 , M.p.; $243{ }^{\circ} \mathrm{C}$. Anal. calcd. for $\mathrm{C}_{16} \mathrm{H}_{12} \mathrm{Cl}_{2} \mathrm{~N}_{2} \mathrm{O}_{5}$ (383): C, 52.06; $\mathrm{H}$, 7.13; $\mathrm{N}, 6.39 \%$. Found: C, 52.12; $\mathrm{H}, 7.21 ; \mathrm{N}$, $6.58 \%$. IR ( $\mathrm{KBr}$ in $\left.\mathrm{cm}^{-1}\right): 3790$ (Ar-OH Str); 3330 (NH Str); 2972 (Ar-CH Str); 1726 (C=O Str); 1591 (CONH Str); 1488 (CH-N Str); 761 (C-Cl Str). ${ }^{1} \mathrm{H}$ NMR (DMSO, $\delta$ ppm): 7.12$8.48(\mathrm{~m}, 6 \mathrm{H}, \mathrm{Ar}-\mathrm{H}), 7.7(\mathrm{~s}, 1 \mathrm{H}, \mathrm{CH}-\mathrm{Cl}), 5.3(\mathrm{~s}$, $1 \mathrm{H}, \mathrm{CH}-\mathrm{N}), 4.8(\mathrm{~s}, 1 \mathrm{H}, \mathrm{NH}), 2.83(\mathrm{~s}, 3 \mathrm{H}$, $3(\mathrm{OH}))$. MS $(\mathrm{m} / \mathrm{z}): 385\left(\mathrm{M}^{+1}\right)$.

(3R, 4S)-3-Chloro-4-(4-chloro phenyl)-N-(3,4,5-trihydroxy benzamido)-2-azetidinone (3g): Yield: $63 \%$, Colour: Pale yellow, $R_{f}$ value: 0.41 , M.p.; $198{ }^{\circ} \mathrm{C}$. Anal. calcd. for $\mathrm{C}_{16} \mathrm{H}_{12} \mathrm{Cl}_{2} \mathrm{~N}_{2} \mathrm{O}_{5}$.(382): $\mathrm{C}, 52.06 ; \mathrm{H}, 7.13 ; \mathrm{N}$, $6.39 \%$. Found: C, 52.12; H, 7.21; N, $6.58 \%$. IR ((KBr in $\left.\mathrm{cm}^{-1}\right)$ : 3790 (Ar-OH Str); $3330(\mathrm{NH}$ Str); 2975 (Ar-CH Str); 1732 (C=O Str); 1592 (CONH Str); 1479 (CH-N Str); 761 (C-Cl Str). ${ }^{1} \mathrm{H}$ NMR (DMSO, $\left.\delta \mathrm{ppm}\right): 7.12-8.48(\mathrm{~m}, 6 \mathrm{H}$, $\operatorname{Ar}-\mathrm{H}), 7.7(\mathrm{~s}, 1 \mathrm{H}, \mathrm{CH}-\mathrm{Cl}), 5.3(\mathrm{~s}, 1 \mathrm{H}, \mathrm{CH}-\mathrm{N})$, $4.8(\mathrm{~s}, 1 \mathrm{H}, \mathrm{NH}), 2.83(\mathrm{~s}, 3 \mathrm{H}, 3(\mathrm{OH})) . \mathrm{MS}$ $(\mathrm{m} / \mathrm{z}): 383\left(\mathrm{M}^{+1}\right)$.

(3R, 4S)-3-Chloro-4-(2-nitro phenyl)-N-(3,4,5-trihydroxy benzamido)-2-azetidinone (3h): Yield: $78 \%$, Colour: White, $R_{f}$ value: 0.49, M.p.; $215{ }^{\circ} \mathrm{C}$; Anal. calcd. for $\mathrm{C}_{16} \mathrm{H}_{12} \mathrm{ClN}_{3} \mathrm{O}_{7}$ (393): C, 50.83; $\mathrm{H}, 6.96 ; \mathrm{N}$, $9.36 \%$. Found: C, 50.55; H, 6.91; N, 9.29\%. IR $\left(\mathrm{KBr}\right.$ in cm$\left.{ }^{-1}\right): 3790$ (Ar-OH Str); $3469(\mathrm{NH}$ Str); 2974 (Ar-CH Str); 1731 (C=O Str); 1598 (CONH Str); 1547 (C-NO 2 Str), 1483 (CH-N Str); 752 (C-Cl Str). ${ }^{1} \mathrm{H}$ NMR (DMSO, $\delta$ 
ppm): 7.23-8.51 (m, 6H, Ar-H), $7.11(\mathrm{~s}, 1 \mathrm{H}$, $\mathrm{CH}-\mathrm{Cl}), 5.63$ (s, 1H, CH-N), $4.49(\mathrm{~s}, 1 \mathrm{H}, \mathrm{NH})$ $2.63(\mathrm{~s}, 3 \mathrm{H}, 3(\mathrm{OH}))$. MS (m/z): $394\left(\mathrm{M}^{+1}\right)$.

(3R, 4S)-3-Chloro-4-(3-nitro phenyl)-N-(3,4,5-trihydroxy benzamido)-2-azetidinone (3i): Yield: $73 \%$, Colour: White, $\mathbf{R}_{\mathrm{f}}$ value: 0.75 , M.p.; $224{ }^{0} \mathrm{C}$. Anal. calcd. for $\mathrm{C}_{16} \mathrm{H}_{12} \mathrm{CIN}_{3} \mathrm{O}_{7}(393): \mathrm{C}, 50.83 ; \mathrm{H}, 6.96 ; \mathrm{N}$, $9.36 \%$. Found: C, 50.47; H, 6.83; N, $9.31 \%$. IR ( $\mathrm{KBr}$ in $\left.\mathrm{cm}^{-1}\right)$ : 3369 (Ar-OH Str); 3457 (NH Str); 2978 (Ar-CH Str); 1745 (C=O Str); 1606 (CONH Str); 1533 (C-NO 2 Str); 1481 ( $\mathrm{CH}-\mathrm{N}$ Str); 759 (C-Cl Str). ${ }^{1} \mathrm{H}$ NMR (DMSO, $\delta$ ppm): 7.41-8.69 (m, 6H, Ar-H), 7.41 (s, 1H, CH-Cl), $5.23(\mathrm{~s}, 1 \mathrm{H}, \mathrm{CH}-\mathrm{N}), 4.52(\mathrm{~s}, 1 \mathrm{H}, \mathrm{NH}), 2.56(\mathrm{~s}$, $3 \mathrm{H}, 3(\mathrm{OH})) \cdot \mathrm{MS}(\mathrm{m} / \mathrm{z}): 394\left(\mathrm{M}^{+1}\right)$.

(3R, 4S)-3-Chloro-4-(4-nitro phenyl)-N-(3,4,5-trihydroxy benzamido)-2-azetidinone (3j): Yield: $66 \%$, Colour: White, $\mathrm{R}_{\mathrm{f}}$ value: 0.80 , M.p.; $231{ }^{0} \mathrm{C}$. Anal.calcd. for $\mathrm{C}_{16} \mathrm{H}_{12} \mathrm{CIN}_{3} \mathrm{O}_{7}(393): \mathrm{C}, 50.83 ; \mathrm{H}, 6.96 ; \mathrm{N}$, $9.36 \%$. Found: C, 50.51; H, 6.67; N, $9.20 \%$. IR ( $\mathrm{KBr}$ in $\left.\mathrm{cm}^{-1}\right)$ : 3330 (Ar-OH Str); 3462 (NH Str); 2976 (Ar-CH Str); 1745 (C=O Str); 1619 (CONH Str); 1548 (C-NO 2 Str); 1477 (CH-N Str); 751 (C-Cl Str). ${ }^{1} \mathrm{H}$ NMR (DMSO, $\delta$ ppm): 7.42-8.87 (m, 6H, Ar-H), $7.63(\mathrm{~s}, 1 \mathrm{H}, \mathrm{CH}-\mathrm{Cl})$, $5.55(\mathrm{~s}, 1 \mathrm{H}, \mathrm{CH}-\mathrm{N}), 4.59(\mathrm{~s}, 1 \mathrm{H}, \mathrm{NH}), 2.34(\mathrm{~s}$, $3 \mathrm{H}, 3(\mathrm{OH}))$. MS (m/z): $394\left(\mathrm{M}^{+1}\right)$.

(3R, 4S)-3-Chloro-4-(4-dimethyl amino phenyl)-N-(-3,4,5-trihydroxy benzamido)-2azetidinone (3k): Yield: $61 \%$, Colour: White, $\mathrm{R}_{\mathrm{f}}$ value: 0.66 , M.p.; $234{ }^{\circ} \mathrm{C}$. Anal. calcd. for $\mathrm{C}_{18} \mathrm{H}_{18} \mathrm{CIN}_{3} \mathrm{O}_{5}(391): \mathrm{C}, 56.43 ; \mathrm{H}, 8.34 ; \mathrm{N}$, $9.40 \%$. Found: C, 56.31; H, 8.26; N, 9.35 \%. IR ( $\mathrm{KBr}$ in $\left.\mathrm{cm}^{-1}\right): 3685$ (Ar-OH Str); 3340 (NH Str); 2974 (Ar-CH Str); 1755 (C=O Str); 1678 (CONH Str); 1472 (CH-N Str); 1301 (N$\mathrm{CH}_{3}$ Str); 759 (C-Cl Str). ${ }^{1} \mathrm{H}$ NMR (DMSO, $\delta$ ppm): 7.33-8.78 (m, 6H, Ar-H), $7.32(\mathrm{~s}, 1 \mathrm{H}$, $\mathrm{CH}-\mathrm{Cl}), 5.57$ (s, 1H, CH-N), $4.81(\mathrm{~s}, 1 \mathrm{H}, \mathrm{NH})$, $2.9\left(\mathrm{~s}, 6 \mathrm{H}, \mathrm{N}\left(\mathrm{CH}_{3}\right)_{2}\right), 2.69(\mathrm{~s}, 3 \mathrm{H}, 3(\mathrm{OH})) . \mathrm{MS}$ $(\mathrm{m} / \mathrm{z}): 392\left(\mathrm{M}^{+1}\right)$.

(3R, 4S)-3-Chloro-4-(3,4,5-trimethoxy phenyl)-N-(-3,4,5-trihydroxy benzamido)-2azetidinone (3I): Yield: 69\%, Colour: Pale brown, $R_{f}$ value: 0.71 , M.p.; $241^{\circ} \mathrm{C}$; Anal. calcd. for $\mathrm{C}_{19} \mathrm{H}_{19} \mathrm{CIN}_{2} \mathrm{O}_{8}(438): \mathrm{C}, 53.49 ; \mathrm{H}$, $7.75 ; \mathrm{N}, 5.67 \%$. Found: C, 53.51; H, 7.82; N, $5.65 \%$. IR ( $\mathrm{KBr}$ in $\left.\mathrm{cm}^{-1}\right): 3470$ (Ar-OH Str); 3298 (NH Str); 2973 (Ar-CH Str); 1724 (C=O Str); 1643 (CONH Str); 1488 (CH-N Str); $1258\left(\mathrm{Ar}-\mathrm{OCH}_{3} \mathrm{Str}\right) ; 756$ (C-Cl Str). ${ }^{1} \mathrm{H}$ NMR (DMSO, $\delta \mathrm{ppm}): 8.21-8.78(\mathrm{~m}, 4 \mathrm{H}, \mathrm{Ar}-\mathrm{H})$, $7.51(\mathrm{~s}, 1 \mathrm{H}, \mathrm{CH}-\mathrm{Cl}), 5.68(\mathrm{~s}, 1 \mathrm{H}, \mathrm{CH}-\mathrm{N}), 4.92$ $(\mathrm{s}, 1 \mathrm{H}, \mathrm{NH}), 3.60\left(\mathrm{~s}, 9 \mathrm{H},\left(\mathrm{OCH}_{3}\right)_{3}\right), 2.47(\mathrm{~s}$, $3 \mathrm{H}, 3(\mathrm{OH})), . \mathrm{MS}(\mathrm{m} / \mathrm{z}): 439\left(\mathrm{M}^{+1}\right)$.

(3R, 4S)-3-Chloro-4-(3,4-dimethoxy phenyl)-N-(-3,4,5-trihydroxy benzamido)-2azetidinone $(3 \mathrm{~m})$ : Yield: $66 \%$, Colour: Pale brown, $\mathrm{R}_{\mathrm{f}}$ value: 0.81, M.p.; $203{ }^{0} \mathrm{C}$; Anal. calcd. for $\mathrm{C}_{18} \mathrm{H}_{17} \mathrm{CIN}_{2} \mathrm{O}_{7}(408)$ : C, 54.36; $\mathrm{H}$, 7.82; N, $6.04 \%$. Found: C, 54.32; H, 7.78; N, $5.98 \%$. IR ( $\mathrm{KBr}$ in $\left.\mathrm{cm}^{-1}\right): 3790$ (Ar-OH Str); 3402 (NH Str); 2977 (Ar-CH Str); 1728 (C=O Str); 1648 (CONH Str); 1475 (CH-N Str); $1256\left(\mathrm{Ar}-\mathrm{OCH}_{3} \mathrm{Str}\right) ; 764$ (C-Cl Str). ${ }^{1} \mathrm{H}$ NMR (DMSO, $\delta$ ppm) 7.25-8.68 (m, 5H, Ar-H), 7.65 (s, $1 \mathrm{H}, \mathrm{CH}-\mathrm{Cl}), 5.43(\mathrm{~s}, 1 \mathrm{H}, \mathrm{CH}-\mathrm{N}), 4.41(\mathrm{~s}$, $1 \mathrm{H}, \mathrm{NH}), 3.32\left(\mathrm{~s}, 6 \mathrm{H},\left(\mathrm{OCH}_{3}\right)_{2}\right), 2.21(\mathrm{~s}, 3 \mathrm{H}$, $3(\mathrm{OH}))$. MS (m/z): $409\left(\mathrm{M}^{+1}\right)$.

(3R, 4S)-3-Chloro-4-(4-methoxy phenyl)-N(-3,4,5-trihydroxy benzamido)-2azetidinone (3n): Yield: 73 \%, Colour: Pale yellow, $R_{f}$ value: 0.48 , M.p.; $218{ }^{\circ} \mathrm{C}$; Anal. calcd.for $\mathrm{C}_{17} \mathrm{H}_{15} \mathrm{ClN}_{2} \mathrm{O}_{6}(378)$ : $\mathrm{C}, 55.36 ; \mathrm{H}$, $7.90 ; \mathrm{N}, 6.49 \%$. Found: C, 55.32; H, 7.88; N, $6.45 \%$. IR ( $\mathrm{KBr}$ in $\left.\mathrm{cm}^{-1}\right): 3769$ (Ar-OH Str); 3402 (NH Str); 2973 (Ar-CH Str); 1732 (C=O Str); 1637 (CONH Str); 1473 (CH-N Str); $1252\left(\mathrm{Ar}-\mathrm{OCH}_{3} \mathrm{Str}\right) ; 750$ (C-Cl Str). ${ }^{1} \mathrm{H}$ NMR (DMSO, $\delta$ ppm): 7.25-8.68(m, 6H, Ar-H), 7.52 (s, $1 \mathrm{H}, \mathrm{CH}-\mathrm{Cl}), 5.76(\mathrm{~s}, 1 \mathrm{H}, \mathrm{CH}-\mathrm{N}), 4.89$ (s, $1 \mathrm{H}, \mathrm{NH}), 3.82\left(\mathrm{~s}, 3 \mathrm{H}, \mathrm{OCH}_{3}\right), 2.43(\mathrm{~s}, 3 \mathrm{H}$, $3(\mathrm{OH}))$. MS (m/z): $379\left(\mathrm{M}^{+1}\right)$.

(3R, 4S)-3-Chloro-4-(4-chloro phenyl)-N-(3,4,5-trihydroxy benzamido)-2-azetidinone (30): Yield: $72 \%$, Colour: Pale yellow, $R_{f}$ value: 0.54 , M.p.; $204{ }^{0} \mathrm{C}$; Anal. calcd. for $\mathrm{C}_{16} \mathrm{H}_{12} \mathrm{Cl}_{2} \mathrm{~N}_{2} \mathrm{O}_{5}$ (382): C, 52.06; $\mathrm{H}, 7.13 ; \mathrm{N}$, 6.39 \%. Found: C, 52.02; H, 7.11; N, 6.41\%. IR (KBr in $\left.\mathrm{cm}^{-1}\right)$ : 3774 (Ar-OH Str); 3400 (NH Str); 2978 (Ar-CH Str); 1721 (C=O Str); 1625 (CONH Str); 1479 (CH-N Str); 764 (C-Cl Str). 
${ }^{1} \mathrm{H}$ NMR (DMSO, $\left.\delta \mathrm{ppm}\right):$ 7.57-8.94 $(\mathrm{m}, 6 \mathrm{H}$, Ar-H), $7.15(\mathrm{~s}, 1 \mathrm{H}, \mathrm{CH}-\mathrm{Cl}), 5.12(\mathrm{~s}, 1 \mathrm{H}, \mathrm{CH}-$ $\mathrm{N}), 4.59(\mathrm{~s}, 1 \mathrm{H}, \mathrm{NH}), 2.45(\mathrm{~s}, 3 \mathrm{H}, 3(\mathrm{OH})), \mathrm{MS}$ $(\mathrm{m} / \mathrm{z}): 383\left(\mathrm{M}^{+1}\right)$.

\section{Evaluation of antimicrobial activity}

The minimum inhibitory concentration (MIC) of the test compounds was determined by broth dilution method $[19,20]$. Each of the test compounds and the standard drugs were dissolved in DMSO at a concentration of 100 $\mu \mathrm{g} / \mathrm{ml}$. Further dilutions of the test compounds and standards in the test medium were prepared at concentrations of 50,25 , $12.5,6.25,3.12,1.56$ and $0.78 \mu \mathrm{g} / \mathrm{ml}$. The bacterial strains of Bacillus subtilis, Staphylococcus aureus, Streptococcus mutans, Escherichia coli, Klebsiella pneumonia and Pseudomonas aeruginosa were maintained on MHA (Muller-Hinton Agar) medium for $24 \mathrm{~h}$ at $37^{\circ} \mathrm{C}$ while fungal strains of Candida albicans and Aspergillus niger were maintained on PDA (Potato Dextrose Agar) medium for $48 \mathrm{~h}$ at $25^{\circ} \mathrm{C}$. The bacterial and fungal suspensions were adjusted with sterile water to a concentration of approximately $103 \mathrm{CFU} / \mathrm{ml}$ and 104 $\mathrm{CFU} / \mathrm{ml}$, respectively. The test compounds and standard drugs, ciprofloxacin and ketoconazole, at various concentrations, were prepared in MHA medium for bacteria and PDA medium for fungi by two-fold serial dilution method and then inoculated and incubated for $24 \mathrm{~h}$ at $37{ }^{\circ} \mathrm{C}$ for bacteria and $48 \mathrm{~h}$ at $25^{\circ} \mathrm{C}$ for fungi. The last tube with no growth of microorganism was recorded as the MIC, expressed in $\mu \mathrm{g} / \mathrm{ml}$.

\section{Evaluation of anti-tubercular activity}

Antitubercular activity was evaluated against Mycobacterium tuberculosis H37 Rv using Microplate alamar blue assay (MABA) method [21,22]. Antitubercular susceptibility test was performed in black, clear-bottomed, 96-well microplates (Packard Instrument Company, Meriden, Conn., USA) in order to minimize background fluorescence. Initial drug dilutions were prepared in dimethylsulfoxide and subsequent two-fold dilutions were performed in $0.1 \mathrm{ml}$ of $7 \mathrm{H} 9 \mathrm{GC}$ media in the microplates. An aliquot $(100 \mu \mathrm{l})$ of $2000 \mathrm{CFU} / \mathrm{ml}$ of $M$. tuberculosis H37 Rv were added to each well of 96-well microtitre plate containing test compounds. Three control well plates containing drug and medium, bacteria and medium, and medium only were also prepared. All microtitre plates were incubated at $37{ }^{\circ} \mathrm{C}$ for seven days. At day 7 of incubation, Alamar Blue dye solution $(20 \mu \mathrm{l}$ Alamar Blue solution and $12.5 \mathrm{ml}$ of 20 $\%$ Tween 80 ) was added to all the wells and the plates re-incubated at $37{ }^{\circ} \mathrm{C}$ for $24 \mathrm{~h}$. Fluorescence was measured in a Victor II multilabel fluorometer (Perkin Elmer Life Sciences Inc., Boston, MA, USA) and MIC was determined.

\section{Statistical anlaysis}

The antimicrobial study was a completely randomized study with three replications. Data were subjected to ANOVA using GraphPad Instat (Version 3.01, University of Kentucky, GTA-50429-259). A significant difference at $p<0.01$ was set for the antimicrobial studies.

\section{RESULTS}

Physicochemical parameters of the synthesized compounds, including color, $R_{f}$ value, and melting point are shown in Table 1 while antimicrobial and antitubercular results are listed in Table 2.

\section{Antimicrobial activity}

Compounds $\mathbf{3 f}, \mathbf{3 g}, \mathbf{3 k}$ and $\mathbf{3 0}$ were active, among the compounds in the series (3a-0), exhibiting antibacterial and antifungal activities as high as those of the reference standards - ciprofloxacin and ketoconazole, respectively. On the other hand, compounds 3a, 3c, 3h, 3i and $\mathbf{3 j}$ exhibited moderate activity.

\section{Antitubercular activity}

Compounds $\mathbf{3 f}, \mathbf{3 g}, \mathbf{3 k}$ and $\mathbf{3 0}$ showed MIC values equivalent to that of the reference standard, isoniazid. Compounds $\mathbf{3 a}, \mathbf{3 c}, \mathbf{3} \mathbf{j}$ 
Ilango \& Arunkumar

Table 1: Physicochemical parameters of the synthesized compounds

\begin{tabular}{|c|c|c|c|c|c|c|}
\hline Code & $\mathbf{R}$ & Colour & Mol.Wt & $\%$ Yield & Melting Pt. & $\mathbf{R}_{\mathrm{f}}$. Value \\
\hline $3 a$ & $\mathrm{H}$ & Dull white & 348.1 & 64 & 221 & 0.63 \\
\hline $3 b$ & $2-\mathrm{OH}$ & Pale yellow & 364.7 & 68 & 198 & 0.78 \\
\hline $3 c$ & $2-\mathrm{OH}-3-\mathrm{OCH}_{3}$ & White & 394.7 & 68 & 217 & 0.72 \\
\hline $3 d$ & $3-\mathrm{OH}$ & White & 364.7 & 62 & 206 & 0.69 \\
\hline $3 e$ & $4-\mathrm{OH}$ & Pale yellow & 364.7 & 72 & 228 & 0.83 \\
\hline $3 f$ & $2-\mathrm{Cl}$ & Light Brown & 382.1 & 71 & 243 & 0.56 \\
\hline $3 g$ & $3-\mathrm{Cl}$ & Pale yellow & 382.1 & 63 & 211 & 0.41 \\
\hline $3 \mathrm{~h}$ & $2-\mathrm{NO}_{2}$ & White & 393.7 & 78 & 215 & 0.49 \\
\hline $3 \mathbf{i}$ & $3-\mathrm{NO}_{2}$ & White & 393.7 & 66 & 224 & 0.75 \\
\hline $3 \mathbf{j}$ & $4-\mathrm{NO}_{2}$ & White & 393.7 & 73 & 231 & 0.80 \\
\hline $3 k$ & $4-\mathrm{N}-\left(\mathrm{CH}_{3}\right)_{2}$ & White & 391.8 & 61 & 234 & 0.66 \\
\hline $3 \mathbf{I}$ & $3,4,5-\left(\mathrm{OCH}_{3}\right)_{3}$ & Pale brown & 438.8 & 69 & 241 & 0.71 \\
\hline $3 m$ & $3,4,-\left(\mathrm{OCH}_{3}\right)_{2}$ & Pale brown & 408.7 & 66 & 203 & 0.81 \\
\hline $3 n$ & $4-\mathrm{OCH}_{3}$ & Pale yellow & 378.7 & 73 & 218 & 0.48 \\
\hline 30 & $4-\mathrm{Cl}$ & Pale yellow & 382.1 & 72 & 204 & 0.54 \\
\hline
\end{tabular}

Table 2: Antimicrobial and antitubercular activities of compounds 3a-o

\begin{tabular}{|c|c|c|c|c|c|c|c|c|c|c|}
\hline \multirow{3}{*}{ Compound } & \multirow{3}{*}{$\mathbf{R}$} & \multicolumn{9}{|c|}{ 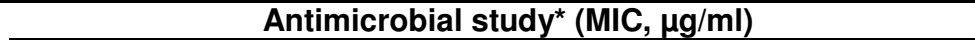 } \\
\hline & & \multicolumn{6}{|c|}{ Antibacterial activity } & \multicolumn{2}{|c|}{$\begin{array}{l}\text { Antifungal } \\
\text { activity }\end{array}$} & \multirow{2}{*}{$\begin{array}{l}\text { Antitu- } \\
\text { bercular } \\
\text { activity } \\
\text { MT }\end{array}$} \\
\hline & & $\overline{B S}$ & SA & SM & EC & KP & PA & $\mathbf{C A}$ & AN & \\
\hline $3 a$ & $-\mathrm{H}$ & 12.5 & 25 & 25 & 12.5 & 25 & 12.5 & 12.5 & 6.25 & 2.8 \\
\hline $3 b$ & $-2-\mathrm{OH}$ & 25 & 12.5 & 25 & 25 & 12.5 & 25 & 25 & 25 & 50.3 \\
\hline $3 c$ & $-2-\mathrm{OH}-3-\mathrm{OCH}_{3}$ & 6.25 & 3.12 & 3.12 & 1.56 & 3.12 & 3.12 & 6.25 & 3.12 & 3.7 \\
\hline $3 d$ & $-3-\mathrm{OH}$ & 25 & 25 & 50 & 12.5 & 12.5 & 12.5 & 12.5 & 25 & 50.3 \\
\hline $3 e$ & $-4-\mathrm{OH}$ & 12.5 & 12.5 & 25 & 25 & 50 & 25 & 12.5 & 25 & 34.2 \\
\hline $3 f$ & $-2-\mathrm{Cl}$ & 6.25 & 12.5 & 3.12 & 6.25 & 3.12 & 6.25 & 3.12 & 1.56 & 0.76 \\
\hline $3 g$ & $-3-\mathrm{Cl}$ & 1.56 & 1.56 & 3.12 & 6.25 & 3.12 & 6.25 & 1.56 & 3.12 & 0.57 \\
\hline $3 \mathrm{~h}$ & $-2-\mathrm{NO}_{2}$ & 25 & 12.5 & 12.5 & 25 & 25 & 12.5 & 25 & 25 & 17.1 \\
\hline $3 i$ & $-3-\mathrm{NO}_{2}$ & 6.25 & 12.5 & 3.12 & 12.5 & 6.25 & 12.5 & 12.5 & 25 & 34.8 \\
\hline $3 j$ & $-4-\mathrm{NO}_{2}$ & 12.5 & 3.12 & 12.5 & 12.5 & 12.5 & 3.12 & 6.25 & 12.5 & 1.2 \\
\hline $3 k$ & $-4-\mathrm{N}-\left(\mathrm{CH}_{3}\right)_{2}$ & 3.12 & 3.12 & 1.56 & 1.56 & 3.12 & 3.12 & 1.56 & 3.12 & 0.62 \\
\hline 3। & $-3,4,5-\left(\mathrm{OCH}_{3}\right)_{3}$ & $>50$ & 25 & $>50$ & 25 & $>50$ & 25 & 25 & 25 & 23.4 \\
\hline $3 m$ & $-3,4,-\left(\mathrm{OCH}_{3}\right)_{2}$ & $>50$ & $>50$ & $>50$ & 25 & 25 & $>50$ & 25 & 25 & 31.4 \\
\hline $3 n$ & $-4-\mathrm{OCH}_{3}$ & $>50$ & 25 & 25 & $>50$ & $>50$ & $>50$ & $>50$ & $>50$ & 2.8 \\
\hline & $-4-\mathrm{Cl}$ & 3.12 & 6.25 & 3.12 & 6.25 & 3.12 & 12.5 & 6.25 & 3.12 & 0.83 \\
\hline Ciprofloxacin & & 1.56 & 3.12 & 3.12 & 1.56 & 3.12 & 1.56 & NA & NA & NA \\
\hline Ketoconazole & & NA & NA & NA & NA & NA & NA & 1.56 & 3.12 & NA \\
\hline Isoniazid & & NA & NA & NA & NA & NA & NA & NA & NA & 0.56 \\
\hline
\end{tabular}

Key: * Mean values $(\mathrm{n}=3)$; BS - Bacillus subtilis; SA - Staphylococcus aureus; SM - Streptococcus mutants; EC Escherichia coli; KP - Klebsiella pneumoniae; PA - Pseudomonas aeruginosa; CA - Candida albicans; AN - Aspergillus niger, MT - Mycobacterium tuberculosis; NA - Not Applicable 
and $3 n$ exhibited moderate activity, while other compounds were less active. The antitubercular activity results correlated well with those of antimicrobial activity.

\section{DISCUSSION}

The monocyclic 2-azetidinones, carbapenams and monobactams are active only against Gram-negative bacteria [23]. The synthesized compounds, however, showed about the same level of activity against both Gram-positive and Gram-negative bacteria. The results of antimicrobial activity and antitubercular activity revealed that compounds $\mathbf{3 f}, \mathbf{3 g}$, and $\mathbf{3 o}$ which have chloro substitution (electron withdrawing group) and 3k which has para dimethyl amino (bulkier group) enhanced the activity of the azetidinones while the other groups, such as hydroxy, methoxy and nitro groups did not influence the activity. Among the chloro substituted compounds (3f, $\mathbf{3 g}$, and $\mathbf{3 0}$ ) compound $\mathbf{3 g}$, which has a chloro group at meta position, exhibited the highest activity in the series. This suggests that electron withdrawing groups and bulkier groups are responsible for the activity. It is interesting to note that some of the currently used antitubercular drugs, such as pyrazinamide, isoniazid and ethionamide, also possess electron withdrawing groups as its pharmacophore. Further studies are going on in our laboratory to establish the quantitative structure activity relationship (QSAR) of the series.

\section{CONCLUSION}

This study indicates that the antimicrobial and antitubercular activities of the synthesized compounds can be attributed to the presence of $2-\mathrm{Cl}, 3-\mathrm{Cl}, 4-\mathrm{Cl}$ and $4-\mathrm{N}-\left(\mathrm{CH}_{3}\right)_{2}$ in phenyl ring at the fourth position of the azetidinone nucleus. The findings of this work should be helpful to medicinal chemists involved in further drug development in this field.

\section{ACKNOWLEDGEMENT}

The authors are thankful to Dr R Shivakumar, Pro-Vice Chancellor, SRM University, Chennai, India and Dr KS Lakshmi, Dean, College of Pharmacy, SRM University, Chennai for making available the facilities used to carry out this work. We also express our profound gratitude to Dr. Ajaykumar for assistance in performing antiubercular tests.

\section{REFERENCES}

1. Frieden TR, Sterling TR, Munsiff SS, Watt CJ, Dye C. Tuberculosis. Lancet 2003; 362: 887-899.

2. Angela GK. Research advances: Onions battle osteoporosis; New weapon in war on TB; smokers beware: Study shows increased cadmium levels in the brain may cause severe neurological disorders. J Chem Educ 2005; 82: 1114-1115

3. Alland D, Kalkut GE, Moss ARN. Genotypic analysis of Mycobacterium tuberculosis in two distinct populations using molecular beacons: Implications for rapid susceptibility testing. N Engl J Med 1994; 330:1710-1715.

4. Davies PDO, Yew WW, Ganguly D, Smoking and tuberculosis: the epidemiological association and pathogenesis. Trans $R$ Soc Trop Med Hyg 2006; 100: 291-298.

5. Whalen C, Horsburgh CR, Hom D. Accelerated course of human immunodeficiency virus infection after tuberculosis. Am J Respir Crit Care Med 1995; 151: 129-135.

6. Sriram D, Yogeeswari $P$, Madhu K. Synthesis and in-vitro antimycobacterial activity of some isonicotinyl hydrazones. Bioorg Med Chem Lett 2005; 15: 4502-4505.

7. Gosling RD, Uiso LO, Sam NE, Bongard E, Kanduma EG, Nyindo M, Morris RW, Gillespie SH. Global Alliance for TB Drug Development: Scientific blueprint for tuberculosis drug development. Tuberculosis 2001; 81: 1-52.

8. Wong CS, Palmer GS, Cynamon MH. In-vitro susceptibility of Mycobacterium tuberculosis, Mycobacterium bovis and Mycobacterium kansasii to Amoxycillin and Ticarcillin in combination with Clavulanic acid. J Antimicrob Chemother 1988; 2: 863-866.

9. Sohi KK, Mittal N, Hundal MK, Khanduja KL. Gallic acid, an antioxidant exhibits antiapoptotic potential in normal human lymphocytes. J Nutr Sci Vitaminol 2003; 49: 221-227.

10. Krogh R, Yunes RA, Andricopulo AD. Structureactivity relationships for the analgesic activity of gallic acid derivatives. Farmaco 2000; 55: $730-735$

11. Anchana $C$, Aphiwat $T$, Jeremy $D$, Nuansri $R$. Antimicrobial gallic acid from Caesalpinia 
mimosoides. Food Chem 2007; 100: 1044 1048.

12. Sakaguchi $N$, Inoue $M$, Isuzugawa $K$, Ogihara $Y$, Hosaka K. Antiproliferative constituents in the plant seeds of Rhynchosia volubilis. Biol Pharm Bull 1999; 22: 471- 475.

13. Nagendra $P K$, Yang $B$, Yang $S$, Yulong $C$, ouming $Z$, Ashraf $M$, Jiang $Y$. Identification of phenolic compounds and appraisal of antioxidant and antityrosinase activities from litchi (Litchi sinensis Sonn.) seeds. Food Chem 2009; 116: $1-7$.

14. Clader JW. Ezetimibe and other azetidinone cholesterol absorption inhibitors. Curr Top Med Chem 2005; 5: 243-256.

15. Gurupadayya BM, Gopal M, Padmashali $B$, Manohara YN. Synthesis and pharmacological evaluation of azetidin-2-ones and thiazolidin-4ones encompassing benzothiazole. Indian $\mathrm{J}$ Pharm Sci 2008; 70: 572-577.

16. Andrew JW, Marvin JM. $\beta$-Lactams in synthesis: short synthesis of cobactin analogs Tetrahedron Lett 2007; 48: 5103-5105.

17. Vijaykumar MM, Nagaraja $T$, Shameer $H$, Jayachandran E, Sreenivasa G. Synthesis, characterization and antimicrobial activity of new N-substituted-3-chloro-2- azetidinones. Arch Pharm Sci Res 2009; 1: 31-39.

18. Patel HS, Patel VK. Synthesis of some new N-[4-N(2,4-dichloro phenyl amino sulfonyl phenyl)-3chloro-4-phenyl azetidin-2-ones]. Oriental $J$ Chem 2002; 18: 513-516.

19. Okusa PN, Penge M, Devesschouwer M, Duez PJ. Direct and indirect antimicrobial effects and antioxidant activity of Cordia gilletii. $J$ Ethnopharmacol 2007; 112: 476-483.

20. Burt SA, Reinders RD. Antibacterial activity of selected plant essential oils against Escherichia coli. Lett Appl Microbiol 2003; 36: 162-169.

21. Collins EA, Franzblow SG. Microplate alamar blue assay versus BACTEC 460 system for highthroughput screening of compounds against Mycobacterium tuberculosis and Mycobacterium avium. Antimicrob Agents Chemother 1997; 41: 1004-1009.

22. Enayat IA, Ashraf HA. Synthesis and antitubercular activity of 6-chloro (unsubstituted)- 2-methoxy9-substituted acridine derivatives. Arch Pharm Res 2004; 27: 713-719.

23 Christopher MC, Richard BS. Monocyclic $\beta$-lactam antibiotics. Med Res Rev 1984; 4: 1-24. 